\title{
REMOTE SENSING FOR A LINING INTEGRATED ACTIVE STRUCTURAL ACOUSTIC CONTROL SYSTEM
}

\author{
Malte Misol* AND Stephan Algermissen $^{\dagger}$ \\ Institute of Composite Structures and Adaptive Systems \\ German Aerospace Center (DLR) \\ Braunschweig, Germany \\ e-mail: *malte.misol@dlr.de, ‘'stephan.algermissen@dlr.de \\ web page: http://www.dlr.de/fa/en
}

Key words: active noise control, aircraft, lining, remote microphone technique

\begin{abstract}
In the framework of the EU project ACASIAS an aircraft sidewall panel (lining) with structurally integrated actuators and sensors is developed. Each lining has a digital unit which samples the sensor signals, performs filtering operations and supplies the actuators with control signals. The whole system makes up an active structural acoustic control system aiming at the reduction of low-frequency multi-tonal aircraft interior noise. The novelty of this approach compared to past implementations of active noise control (ANC) systems in aircraft is its modularity. Each so-called smart lining is autonomous in the sense that it processes only structural sensor data from its own integrated sensors. The use of external microphones for error sensing is avoided because this conflicts with the modularity of the smart lining. Hence, one important design task is the replacement of the physical error microphones by the integrated structural sensors and an acoustic filter (observer) running on the digital unit. This method, which is called the remote microphone technique for active control, has never been applied to an aircraft interior structure so far. The detailed design of the smart lining module comprises several steps which are taken within work package 3 of the ACASIAS project. Experimental data of an aircraft typical double panel system is captured in a sound transmission loss facility. The system is excited with a loudspeaker array placed directly in front of the fuselage structure. Different acoustic load cases are used for the definition of the sensors and the actuators. A multi-tonal excitation with high sound pressure level is relevant for the actuator dimensioning and a broadband excitation with multiple independent sound sources is relevant for the sensor definition. 19 accelerometers are mounted on the lining and 20 microphones are placed in front of it. All sensor signals are sampled simultaneously for deterministic and broadband load cases. The lining is equipped with two inertial mass actuators which are used for the active control. Measured frequency response functions of actuators at 39 positions are used for the optimization of the actuator locations. The measurement data is also used for the derivation of an observer and for the simulation of a smart lining with remote microphones. In this contribution, the steps undertaken for the detailed design will be described and simulation results of the noise reduction performance of the smart lining with remote microphones will be presented.
\end{abstract}




\section{INTRODUCTION}

The active control of rotor noise in aircraft is an ongoing research topic for more then thirty years. Some of these approaches have been successfully implemented in aircraft (e.g. Saab 2000 or Bombardier Dash 8 Q 400). A common solution uses loudspeakers in the cabin to reduce the interior sound pressure by so-called anti-sound (ANC). Early results of ANC in aircraft are documented by Elliott et al. [1]. An alternative approach is the active structural acoustic control (ASAC) of the fuselage by means of shakers or piezoelectric patch actuators. Early results on ASAC in aircraft are documented by Fuller and Jones [2]. A similar approach for active interior noise reduction uses active trim panels (linings) instead of actuated fuselage structures. Experimental work on aircraft interior noise reduction with active linings is reported by Tran and Mathur [3]. More recent work on this topic is published by Misol et al. [4, 5]. Misol et al. [4] use a serial production Airbus A350 lining augmented with actuators, sensors and an active control system. The experiments are done in a sound transmission loss facility. Full-scale tests in a Dornier Do728 aircraft (on the ground) with two active lining modules are documented in Misol [5]. The acoustic effect of the rotor engines on the fuselage is mimicked by means of a loudspeaker array. A maximum (mean) SPL reduction of $11.3 \mathrm{~dB}(6.8 \mathrm{~dB})$ is achieved in the Do728 cabin in front of the active linings. All of the mentioned active noise control systems have in common that they use microphones as error sensors. These microphones are distributed

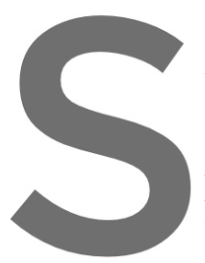
all over the cabin whic ular so-called smart active control proposed by Roure and Albarre phones. The RMT uses accelerometers on the within the controller as
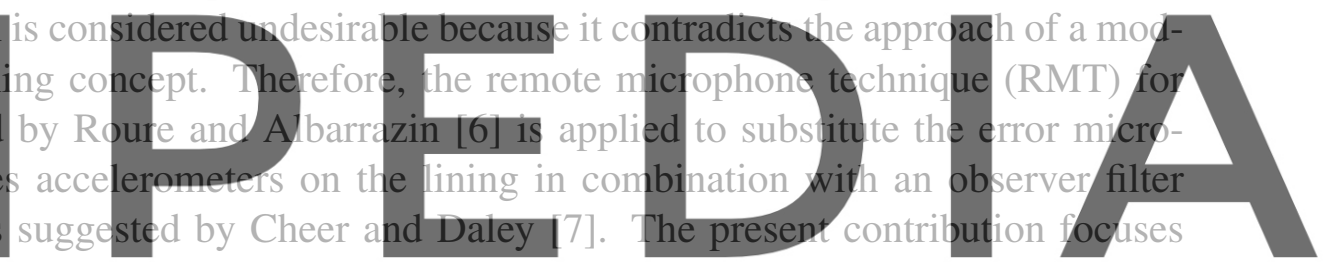

on the noise reduction capability of an active lining system using the RMT. Simulations are

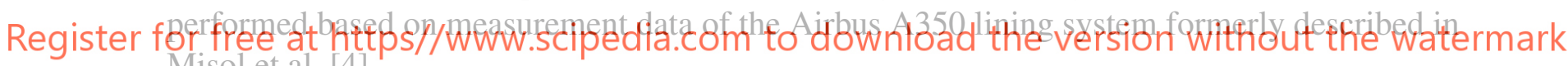
Misol et al. [4].

\section{EXPERIMENTAL SETUP}

Figure 1 shows a scheme of the experimental setup in a sound transmission loss facility. The loudspeaker array (LSA) is placed in front of the fuselage structure with approx. $0.15 \mathrm{~m}$ offset. Out of the 112 loudspeakers 72 are used (yellow area). Two different acoustic load cases are considered. First, the lowest five harmonics of a counter rotation open rotor (CROR) engine $(119.4 \mathrm{~Hz}, 149.2 \mathrm{~Hz}, 268.6 \mathrm{~Hz}, 388 \mathrm{~Hz}$ and $417.9 \mathrm{~Hz}$ ) are synthesized (see Algermissen et al. [8]) and second, all 72 loudspeakers are controlled with uncorrelated bandlimited white noise signals. The test specimen consisting of a carbon fiber reinforced plastics (CFRP) fuselage structure and a coupled lining is mounted in the test opening of the facility. Figure 2 shows the experimental setup seen from the semi-anechoic room. From a total of 19 accelerometers, 16 are selected as potential remote sensors for the RMT (the discarded ones are marked with an X). Although 35 out of 39 different actuator positions are considered (the discarded ones are marked with an X), the acoustic frequency response function (FRF) is available only for actuators 1 and 12. For the other actuators the structural FRF to the grid points (grey dots on the lining in Fig. 2) are known from scanning laser vibrometer measurement. If the grid points are assumed 


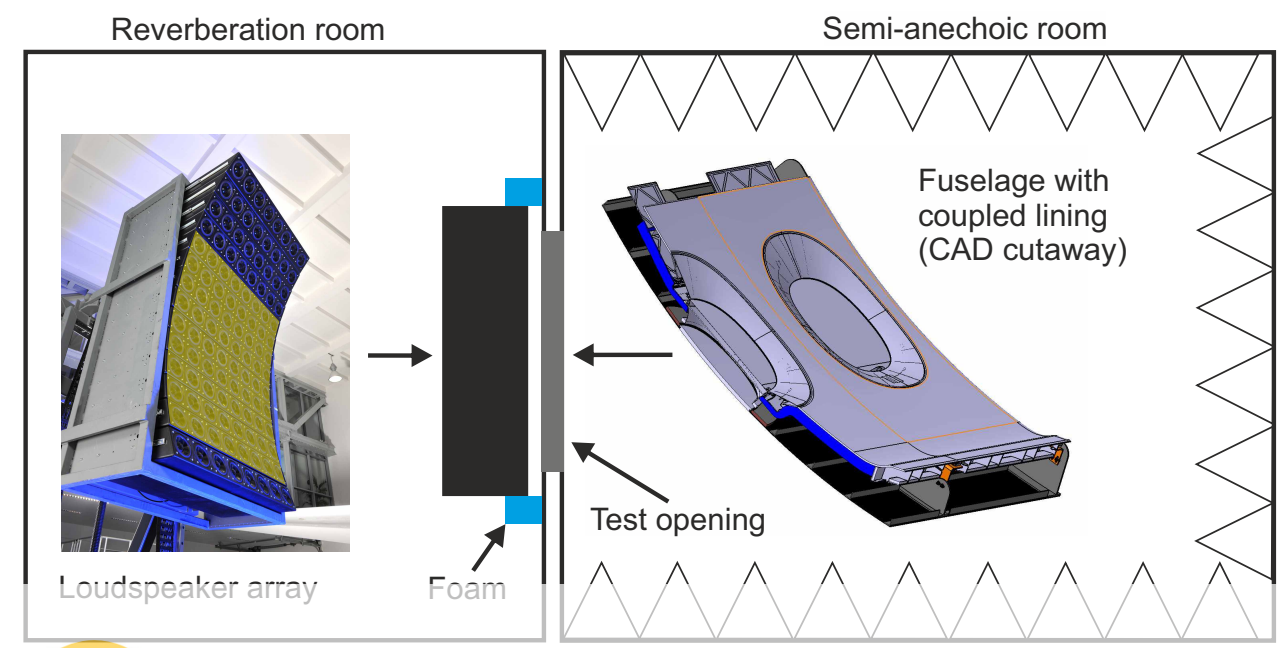

Figure 1: Top view schematic of the experimental setup in the transmission loss facility showing the loudspeaker array in the reverberation room and the fuselage-lining-system mounted in the test opening between the reverberation and the semi anechoic room. The selected loudspeakers are highlighted in yellow. The gap between the fuselage structure and the loudspeaker array is proofed with foam to reduce reverberation.
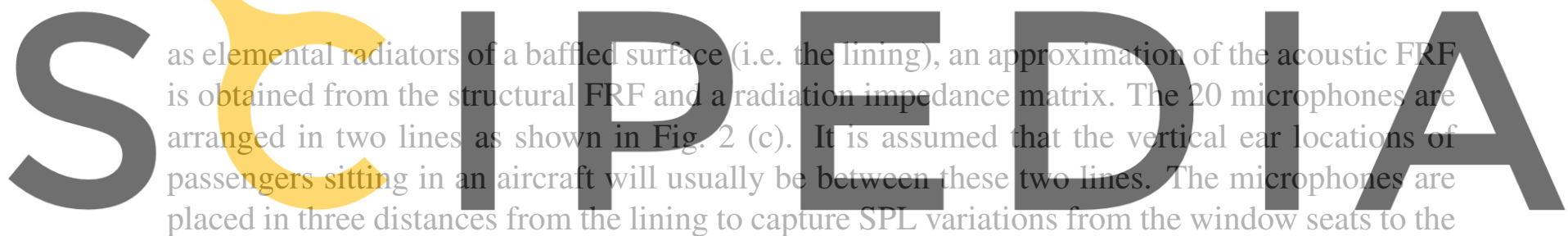
placed in three distances from the lining to capture SPL variations f

Register for free at https//WwW.scipedia.com to download the version without the watermark

\section{SENSORS AND ACTUATORS}

The defining load case for the sensors is the broadband excitation. In that case, an accurate pressure estimate requires a sufficient number of remote sensors. A quantification of sufficiency is provided by the multiple coherence function. Therefore, it is applied as a metric to, firstly, define the required number and, secondly, the best combination of remote sensors. In Eq. (1), $\mathbf{x}$ is the vector of accelerometer signals and $y$ is the sound pressure at one of the 60 microphone positions. The spectra of these signals are denoted by $\mathbf{X}$ and $Y$.

$$
\bar{C}=\frac{1}{n_{2}-n_{1}+1} \sum_{k=n_{1}}^{n_{2}} \frac{\mathbf{S}_{x y}(k) \mathbf{S}_{x x}^{-1}(k) \mathbf{S}_{x y}^{H}(k)}{S_{y y}(k)}
$$

The multiple coherence function is averaged twice. First, over the discrete frequency $k$ (from $50 \mathrm{~Hz}$ to $500 \mathrm{~Hz}$ ) leading to $\bar{C}$ as given by Eq. (1) and second, over all 60 microphone positions leading to $\bar{C}_{a v g}$. The power spectral densities (PSD) are defined as: $\mathbf{S}_{x y}=E\left\{Y \mathbf{X}^{H}\right\}$, $\mathbf{S}_{x x}=E\left\{\mathbf{X X}^{H}\right\}$ and $S_{y y}=E\left\{Y Y^{H}\right\}$ with $E\{\cdot\}$ being the expectation operator. A target value of $\bar{C}_{\text {avg }}=0.8$ is chosen. This corresponds to an explanation of roughly $90 \%$ of the sound pressure amplitude by the remote sensors. The calculation of $\bar{C}$ for different sensor configurations 


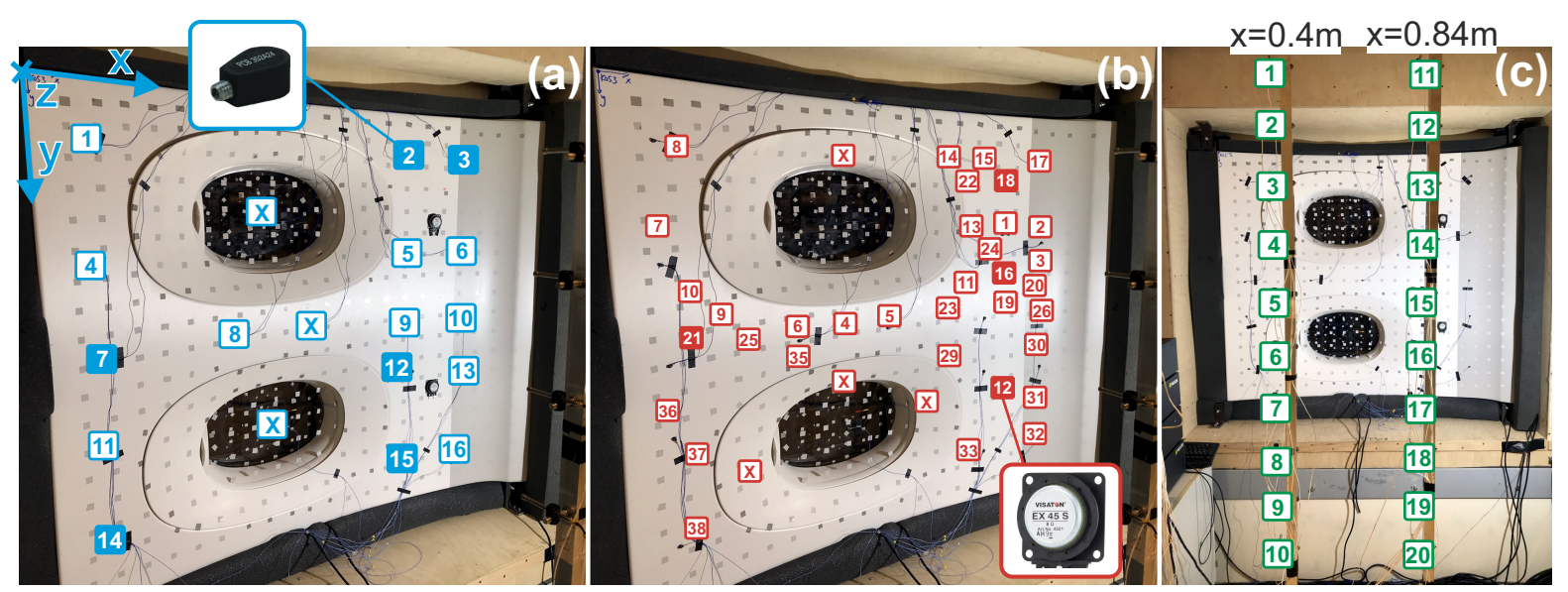

Figure 2: Experimental setup in the transmission loss facility seen from the semi anechoic room. The accelerometers are indicated in blue, the actuators in red and the microphones in green. The accelerometers chosen for the observer of the remote microphone method are 2, 3, 7, 12, 14, 15. The selected actuators are 12, 16, 18 and 21. The microphones are positioned $\mathrm{z}=-0.5 \mathrm{~m}, \mathrm{z}=-1 \mathrm{~m}$ or $\mathrm{z}=-1.5 \mathrm{~m}$ in front of the lining along two vertical lines at $\mathrm{x}=0.4 \mathrm{~m}$ and $\mathrm{x}=0.84 \mathrm{~m}$ spanning two surfaces used for control performance evaluation. Discarded transducer positions are marked with $\mathrm{X}$.
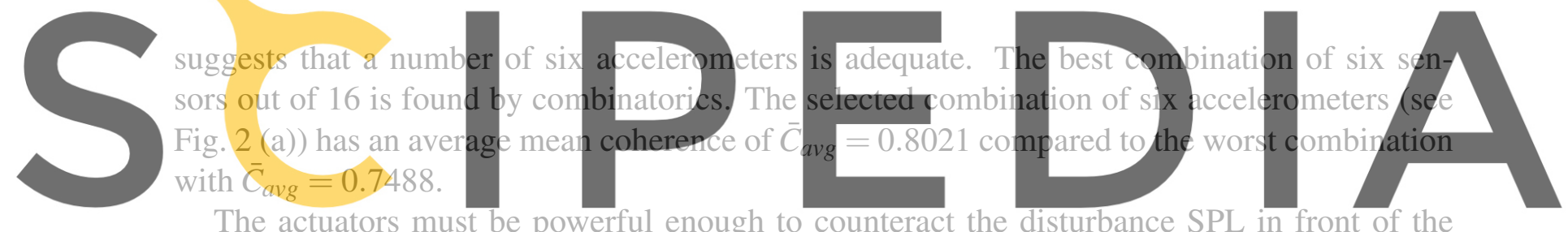

The actuators must be powerful enough to counteract the disturbance SPL in front of the Register for free. A CROR engine is assumed to produce an exterior SPI of $130 \mathrm{~dB}$ in front of the fuselage These high SPL can not be realized With the LSA. The SPL produced in the laboratory by the

LSA in front of the fuselage is roughly $113 \mathrm{~dB}$. Therefore, the selected actuator configuration must provide a stroke excess of at least $17 \mathrm{~dB}$. In the experiments two actuators of the type Visaton ${ }^{\circledR}$ EX45S are attached to the lining at positions 1 and 12 (see Fig. 2 (b)). The calculation of the control voltage $\mathbf{U}$ needed to counteract the CROR induced pressure field $\mathbf{D}_{a}$ in front of the lining results from Eq. (2).

$$
\mathbf{D}_{a}(k) \stackrel{!}{=} \hat{\mathbf{G}}_{a}(k) \mathbf{U}(k) \rightarrow \mathbf{U}(k)=\left[\hat{\mathbf{G}}_{a}(k)^{H} \hat{\mathbf{G}}_{a}(k)\right]^{-1} \hat{\mathbf{G}}_{a}(k)^{H} \mathbf{D}_{a}(k)
$$

The acoustic FRF of the two actuators are denoted with $\hat{\mathbf{G}}_{a}$. The inverse Fourier transform of $\mathbf{U}$ containing the values from Eq. (2) for the five CROR frequency lines yiels the control voltage $\mathbf{u}$ in the time domain. The required voltage amplitude is $0.924 \mathrm{~V}$ which is far below the maximum value of $8.9 \mathrm{~V}$. Assuming a safety factor of two on the voltage, this corresponds to a actuator stroke margin of $13.7 \mathrm{~dB}$. Since the difference between the real and the laboratory SPL is assumed $17 \mathrm{~dB}$ or more, the number of actuators is doubled resulting in a actuator stroke margin of roughly $20 \mathrm{~dB}$. The 35 actuator positions are ranked according to the amplitudes of the estimated acoustic FRF. The four actuator locations associated with the highest mean values are considered the most suitable. These are the ones at the positions 12, 16, 18 and 21. The 
(a)

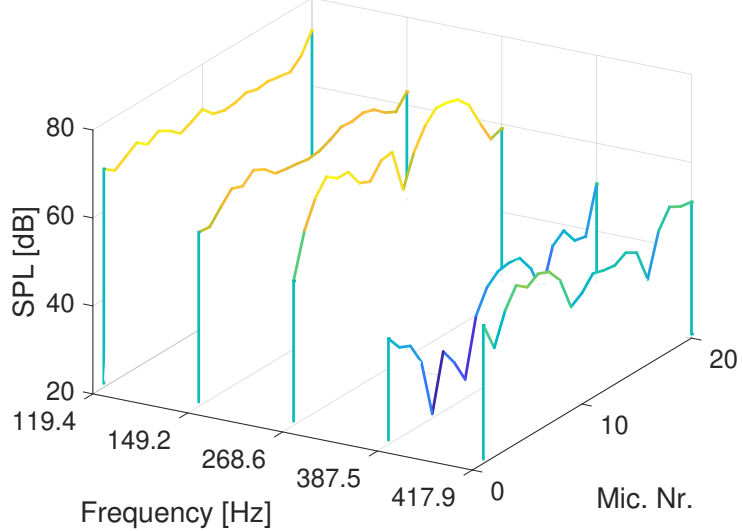

(b)

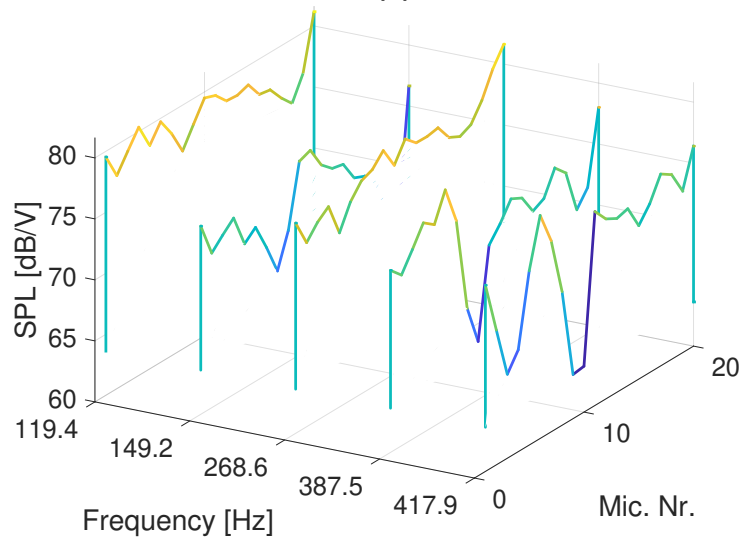

Figure 3: SPL at the CROR frequencies measured by 20 microphones at $\mathrm{z}=-1 \mathrm{~m}$ with the LSA turned on (a) and with actuators 1 and 12 (b).

amplitude difference between the best and the worst location out of 35 is $7.8 \mathrm{~dB}$.

Figure 3 shows the SPL in front of the lining at the CROR frequencies measured by 20 microphones at a distance of one meter. The SPL on the sending side measured between the LSA and the fuselage pandl $78.6 \mathrm{~dB}$ at $387.5 \mathrm{~Hz}$ the theoretical maximu value of the required volt

4 RESULTS

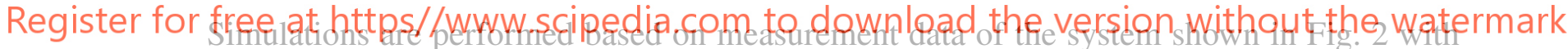
actuators 1 and 12 and remote sensors 2, 3, 7, 12, 14 and 15. The final actuator configuration 12,

16,18 and 21 is not simulated because the exact acoustic FRF are unavailable. It is assumed that the noise reduction performance will improve due to the increased number of control sources. Results are given for two different virtual microphone configurations. Configuration one uses 30 virtual microphones in the plane at $\mathrm{x}=0.4 \mathrm{~m}$ and configuration two uses 30 virtual microphones in the plane at $\mathrm{x}=0.4 \mathrm{~m}$ and 30 virtual microphones in the plane at $\mathrm{x}=0.84 \mathrm{~m}$. The plane dimensions of both configurations are $L_{y} \times L_{z}=1.35 \times 1 \mathrm{~m}^{2}$. Configuration one achieves a mean SPL reduction of $6.4 \mathrm{~dB}(3.8 \mathrm{~dB}(\mathrm{~A}))$ and a maximum SPL reduction of $17 \mathrm{~dB}$ or $(13 \mathrm{~dB}(\mathrm{~A}))$. Configuration two achieves a mean SPL reduction of $7.9 \mathrm{~dB}(5.2 \mathrm{~dB}(\mathrm{~A}))$ and a maximum SPL reduction of $12 \mathrm{~dB}(11 \mathrm{~dB}(\mathrm{~A}))$. Since configuration two uses virtual microphones in both planes, it achieves a more homogenous SPL reduction then configuration one. This is reflected in an increased mean and a decreased maximum SPL reduction. More information on the simulation can be found in Misol [9]. 


\section{CONCLUSIONS}

This contribution describes research activities regarding the detailed design of an active lining module for aircraft interior noise reduction. The derived actuator and sensor configuration is suitable for a CROR engine load case with exterior SPL up to $130 \mathrm{~dB}$. Simulation results show that the active lining reduces the mean SPL by $7.9 \mathrm{~dB}$ or $5.2 \mathrm{~dB}(\mathrm{~A})$ in the controlled volume.

\section{ACKNOWLEDGMENTS}

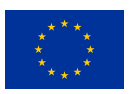

This project has received funding from the European Unions Horizon 2020 research and innovation programme under grant agreement No. 723167.

\section{REFERENCES}

[1] S. J. Elliott, P. A. Nelson, I. M. Stothers, and C. C. Boucher, "In-flight experiments on the active control of propeller-induced cabin noise," Journal of Sound and Vibration, vol. 140, no. 2, pp. 219-238, 1990.

[2] C. R. Fuller and J. D. Jones, "Experiments on reduction of propeller induced interior noise by active control of cylinder vibration," Journal of Sound and Vibration, vol. 112, no. 2, pp. 389-395, Jan. 1987.

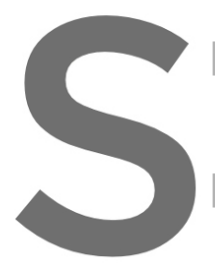

3] B. N. Tran and G.

els," in Proceedings

4] M. Misol, T. Haase

Flugzeugen mit akt
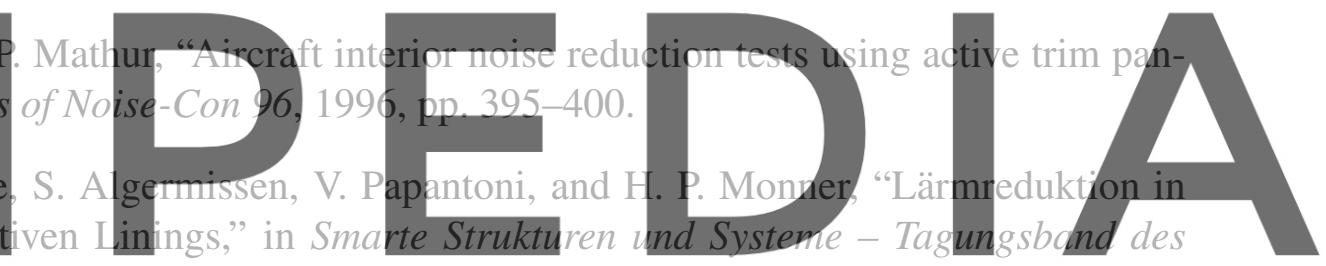

4SMARTS-Symposiums, 2017, pp. 329-339.

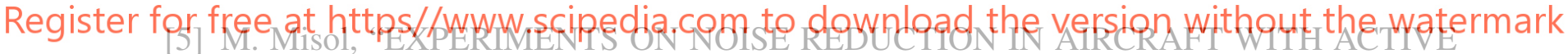
SIDEWALL PANELS," in Proceedings of the 25th International Congress on Sound and Vibration, 2018. [Online]. Available: https://elib.dlr.de/121168/

[6] A. Roure and A. Albarrazin, "The remote microphone technique for active noise control," in PROCEEDINGS OF ACTIVE 99: THE INTERNATIONAL SYMPOSIUM ON ACTIVE CONTROL OF SOUND AND VIBRATION, VOLS 1 \& 2, 1999, pp. 1233-1244.

[7] J. Cheer and S. Daley, "Active structural acoustic control using the remote sensor method," Journal of Physics: Conference Series, vol. 744, no. 1, 2016.

[8] S. Algermissen, S. Meyer, C. Appel, and H. P. Monner, "Experimental synthesis of sound pressure fields for active structural acoustic control testing," Journal of Intelligent Material Systems and Structures, 2013. [Online]. Available: http://jim.sagepub.com/content/early/2013/06/20/1045389X13493361.abstract

[9] M. Misol, "ACTIVE SIDEWALL PANELS WITH REMOTE MICROPHONE TECHNIQUE FOR AIRCRAFT INTERIOR NOISE REDUCTION," in Proceedings of the 26th International Congress on Sound and Vibration, 2019, (accepted for publication). 